\title{
Improvement in Efficiency of Wireless Power Transfer of Magnetic Resonant Coupling Using Magnetoplated Wire
}

\author{
Tsutomu Mizuno ${ }^{1}$, Shintaro Yachi $^{1}$, Akira Kamiya ${ }^{1}$, Daisuke Yamamoto ${ }^{1}$ \\ ${ }^{1}$ Faculty of Engineering, Shinshu University, Nagano 380-8553, Japan
}

\begin{abstract}
Wireless power transfer is expected in the use of an electric vehicle and a chip card. However, it requires a high efficiency and takes a long distance. In this paper, we propose the use of a magnetoplated wire (MPW), which is a copper wire (COW) whose circumference is plated with a magnetic thin film, to improve transmission efficiency. The MPW can reduce resistances due to the proximity effect comparison with the COW. The inner diameter of COW and MPW coils is $d_{\mathrm{i}}=37 \mathrm{~mm}$ and their number of turns is $n=10$. As a result, the resistances of the COW and MPW at the frequency $f=12 \mathrm{MHz}$ are $6.8 \Omega$ and $4.1 \Omega$, respectively, which show a reduction of $40 \%$. The quality factors of the COW and MPW at the frequency $f=12 \mathrm{MHz}$ are 83 and 138, respectively, which show an increase of $66 \%$. The efficiencies of the COW and MPW at a transmission distance of $10 \mathrm{~mm}$ are $69.8 \%$ and $77.7 \%$, respectively, which show an increase of $7.9 \%$.
\end{abstract}

Index Terms - wireless power transfer, magnetic resonant coupling, efficiency, magnetoplated wire, litz wire, quality factor.

\section{INTRODUCTION}

$\mathrm{T}$ he technology of the wireless power transfer of magnetic resonant coupling has already been utilized, for example, in IC cards, and its practical application to portable telephones and electric automobiles is expected [1]. The wireless power transfer of magnetic resonant coupling takes a long distance and can be used in long-distance transmission with a high efficiency [2]. It is necessary to improve the quality factors of transmitting and receiving coils for long-distance and highefficiency transmission. For the improvement in the quality factor characteristic, a litz wire is generally used to decrease the $\mathrm{AC}$ resistance due to the skin effect of a coil [3]. However, the decrease in $\mathrm{AC}$ resistance caused by the proximity effect is difficult, and there is a limit in increasing the quality factor using a litz wire.

Therefore, the authors propose the use of a litz wire (LMW) with a magnetoplated wire (MPW) to improve the transmission efficiency [4]. The MPW is a copper wire (COW) plated with a magnetic thin film. The MPW increases the inductance and decreases the $\mathrm{AC}$ resistance due to the proximity effect. This is because an alternating magnetic flux flows in the magnetic thin film with larger permeability and resistivity than copper. As a result, by the magnetic thin film, the inductance is increased, and the resistance due to the proximity effect is decreased because eddy current loss is reduced [5], [6].

In this paper, the impedance characteristics of coils using litz wires (LCWs) with COWs and LMWs are compared. In addition, the transmission efficiency characteristics of the LCW and LMW coils are compared, and the other following characteristics are described.

(1) Impedance characteristics of the LCW and LMW coils

(2) Transmission efficiency characteristics of the wireless power transfer of magnetic resonant coupling

\section{WIRELESS POWER TRANSFER OF MAGNETIC RESONANT COUPLING USING MAGNETOPLATED WIRE}

Fig. 1 shows the structures of the COW and MPW. The COW has a diameter of $100 \mu \mathrm{m}$ and is plated with insulating films. The MPW is a copper wire with a diameter of $100 \mu \mathrm{m}$ and is plated with magnetic thin films $(\mathrm{Fe}$ and $\mathrm{Ni})$. The thicknesses of the $\mathrm{Fe}$ and $\mathrm{Ni}$ thin films are 0.9 and $0.05 \mu \mathrm{m}$, respectively. The Ni film is plated for ease of soldering.

Fig. 2 shows the structures of the LCW and LMW. Each litz wire is composed of eight wires.

Fig. 3 shows the wireless power transfer of magnetic resonant

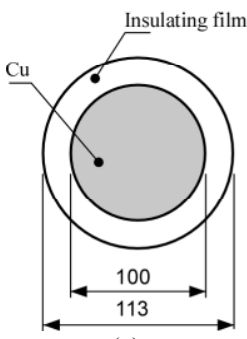

(a)

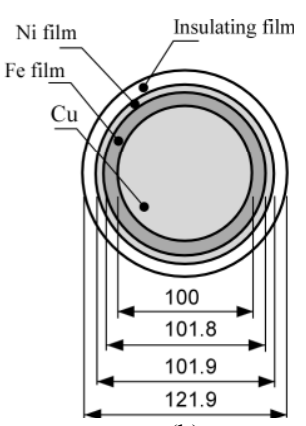

(b)
Fig. 1. Structures of COW and MPW (unit: $\mu \mathrm{m}$ ). (a) COW. (b) MPW.

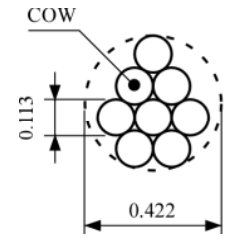

(a)

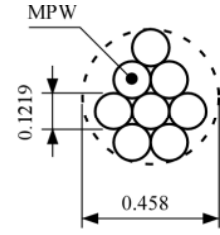

(b)
Fig. 2. Litz wires using COW and MPW (unit: mm). (a) LCW. (b) LMW. 


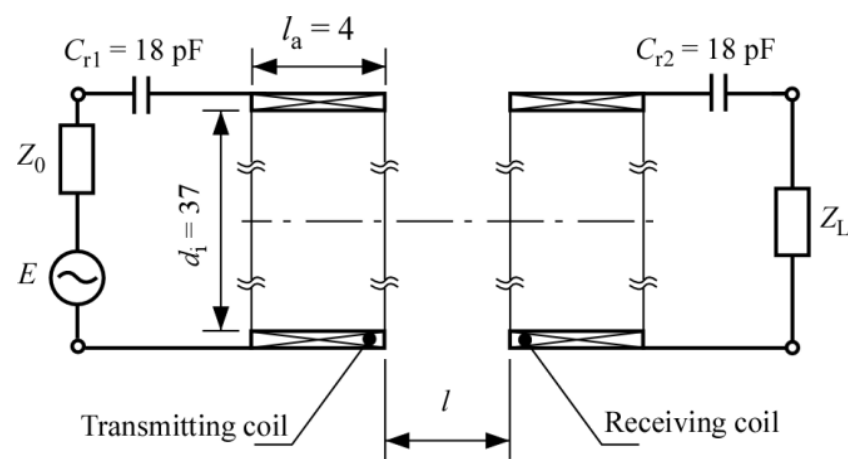

Fig. 3. Wireless power transfer of magnetic resonant coupling (unit: mm).

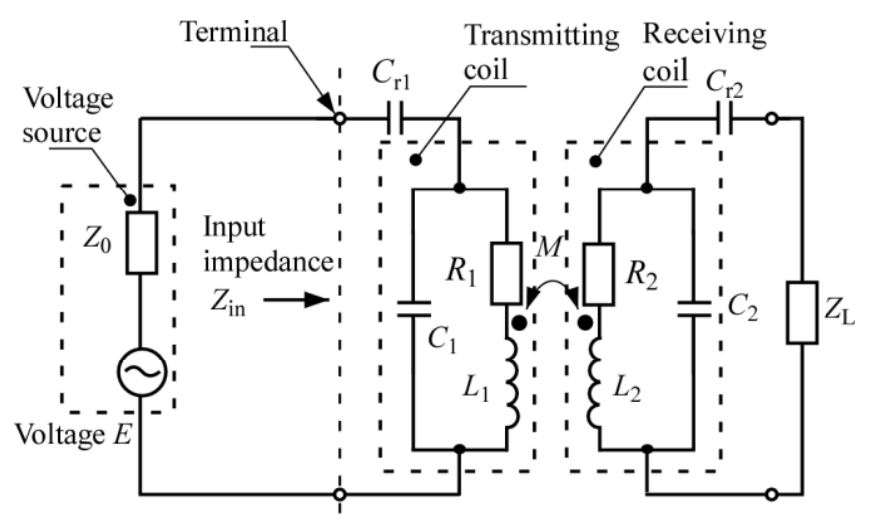

Fig. 4. Equivalent circuit of magnetic resonant coupling.

coupling. The inner diameter of the transmitting and receiving coils is $d_{\mathrm{i}}=37 \mathrm{~mm}$ and their number of turns is $n=10$, and the capacitors $C_{\mathrm{r} 1}=C_{\mathrm{r} 2}=18 \mathrm{pF}$ are connected in series. The coils are air core coils wound closely, and the axial direction length of the LCW and LMW coils is $l_{\mathrm{a}}=4 \mathrm{~mm}$. The transmission efficiency is measured at the transmission distance $l=10 \mathrm{~mm}$ and $18.5 \mathrm{~mm}$.

\section{BASIC CHARACTERISTICS OF COIL}

Fig. 4 shows the equivalent circuit of magnetic field resonant coupling. The transmitting coil is represented by the resistance $R_{1}$, primary inductance $L_{1}$, and stray capacitance $C_{1}$. The receiving coil is represented by the resistance $R_{2}$, secondary inductance $L_{2}$, and stray capacitance $C_{2}$. These coils are coupled at the mutual inductance $M$. The capacitors $C_{\mathrm{r} 1}$ and $C_{\mathrm{r} 2}$ are respectively connected to the transmitting and receiving coils in series. $Z_{0}$ represents the output impedance of power supply, and $Z_{\mathrm{L}}$ load impedance. The transmission efficiency $\eta_{21}$ from the transmitting side to the receiving side is shown by the following equation with the transmission coefficient $S_{21}$ from the transmitting side to the receiving side [7]:

$$
\eta_{21}=\left|S_{21}\right|^{2} \times 100=\left|\frac{2}{\alpha_{1}+\mathrm{j} \beta_{1}}\right|^{2} \times 100
$$

$$
\begin{aligned}
\alpha_{1}= & \frac{1}{\omega^{2} k \sqrt{L_{1} L_{2}} C_{\mathrm{r} 1} C_{\mathrm{r} 2} Q_{1} Q_{2} Z_{0}}\left[Z _ { 0 } \left[\omega ^ { 2 } \left[Q _ { 1 } Q _ { 2 } \left[L_{1} C_{\mathrm{r} 2}\left(C_{\mathrm{r} 1}+C_{1}\right)\right.\right.\right.\right. \\
& \left.+L_{1} C_{\mathrm{r} 1} C_{1}+L_{2} C_{\mathrm{r} 1}\left(C_{\mathrm{r} 2}+C_{2}\right)+L_{2} C_{\mathrm{r} 2} C_{2}\right] \\
& +\omega^{2} L_{1} L_{2}\left\{1-Q_{1} Q_{2}\left(1-k^{2}\right)\right\}\left\{C_{\mathrm{r} 2} C_{2}\left(C_{\mathrm{r} 1}+C_{1}\right)\right. \\
& \left.\left.\left.+C_{\mathrm{r} 1} C_{1}\left(C_{\mathrm{r} 2}+C_{2}\right)\right\}\right]-Q_{1} Q_{2}\left(C_{\mathrm{r} 1}+C_{\mathrm{r} 2}\right)\right]-\omega L_{2} Q_{1}\left(C_{\mathrm{r} 2}+C_{2}\right) \\
& -\omega L_{1} Q_{2}\left(C_{\mathrm{r} 1}+C_{1}\right)+\omega^{3} L_{1} L_{2}\left(C_{\mathrm{r} 1}+C_{1}\right)\left(C_{\mathrm{r} 2}+C_{2}\right)\left(Q_{1}+Q_{2}\right) \\
& +\omega^{3} C_{\mathrm{r} 1} C_{\mathrm{r} 2} Z_{0} Z_{\mathrm{L}}\left\{L_{1} C_{1} Q_{21}+L_{2} C_{2} Q_{1}\right. \\
& \left.\left.-\omega^{2} L_{1} L_{2} C_{1} C_{2}\left(Q_{1}+Q_{2}\right)\right\}\right] \\
\beta_{1}= & \frac{1}{\omega^{3} k \sqrt{L_{1} L_{2}} C_{\mathrm{r} 1} C_{\mathrm{r} 2} Q_{1} Q_{2} Z_{0}}\left[-\omega^{3} Z_{0}\left[\left\{L_{1} Q_{2}\right.\right.\right. \\
& \left.-\omega^{2} L_{1} L_{2} C_{2}\left(Q_{1}+Q_{2}\right)\right\}\left(C_{\mathrm{r} 1}+C_{1}\right) C_{\mathrm{r} 2}+L_{1} C_{1} C_{\mathrm{r} 1} Q_{2} \\
& \left.+\left\{L_{2} Q_{1}-\omega^{2} L_{1} L_{2} C_{1}\left(Q_{1}+Q_{2}\right)\right\}\left(C_{\mathrm{r} 2}+C_{2}\right) C_{\mathrm{r} 1}+L_{2} C_{2} C_{\mathrm{r} 2} Q_{1}\right] \\
& +Q_{1} Q_{2}-\omega^{2} L_{2} Q_{1} Q_{2}\left(C_{\mathrm{r} 2}+C_{2}\right) \\
& -\omega^{2}\left(C_{\mathrm{r} 1}+C_{1}\right)\left[L_{1} Q_{1} Q_{2}+\omega^{2} L_{1} L_{2}\left(C_{\mathrm{r} 2}+C_{2}\right)\left\{1-Q_{1} Q_{2}\left(1-k^{2}\right)\right\}\right] \\
& +\omega^{2} C_{\mathrm{r} 1} C_{\mathrm{r} 2} Z_{0} Z_{\mathrm{L}}\left[\left[\omega^{2} L_{1} C_{1} Q_{1} Q_{2}+L_{2} C_{2} Q_{1} Q_{2}\right.\right. \\
& \left.\left.\left.+\omega^{2} L_{1} L_{2} C_{1} C_{2}\left\{1-Q_{1} Q_{2}\left(1-k^{2}\right)\right\}\right]-Q_{1} Q_{2}\right]\right]
\end{aligned}
$$

where $\alpha_{1}$ and $\beta_{1}$ are the coefficients of the real and imaginary parts of $S_{21}, \omega$ is the angular frequency $(\mathrm{rad} / \mathrm{s}), k$ is the coupling coefficient, $L_{1}$ and $L_{2}$ are the primary and secondary inductances $(\mathrm{H}), C_{\mathrm{r} 1}$ and $C_{\mathrm{r} 2}$ are the capacitances $(\mathrm{F})$ of the transmitting and receiving sides, $Q_{1}$ and $Q_{2}$ are the quality factors of the transmitting and receiving coils, $Z_{0}$ is the output impedance $(\Omega)$ of power supply, $Z_{\mathrm{L}}$ is the load impedance $(\Omega)$, and $C_{1}$ and $C_{2}$ are the stray capacitances (F) of the transmitting and receiving coils, respectively.

Equations (1), (2), and (3) show that the coupling coefficient and the resistance of the coils must increase to improve the efficiency $\eta_{21}$ [7]. The coupling coefficient depends on transmission distance; thus, it decreases with increasing transmission distance. Then, the decrease in the resistance or the increase in quality factor is examined for the improvement in efficiency $\eta_{21}$.

Fig. 5 shows the impedance versus frequency characteristics of the coils. The impedance characteristics were measured with an impedance analyzer (Agilent, 4294A). The resistances of the LCW and LMW coils at the frequency $f=12 \mathrm{MHz}$ were $6.8 \Omega$ and $4.1 \Omega$, respectively; thus, the resistance of the LMW decreases by $40 \%$ compared with that of the LCW. The inductances of the LCW and LMW coils at the frequency $f=$ $12 \mathrm{MHz}$ were 7.47 and $7.57 \mu \mathrm{H}$, respectively; thus, the inductance of the LMW increases by $1.3 \%$ compared with that of the LCW. Therefore, the quality factors of the LCW and LMW coils at the frequency $f=12 \mathrm{MHz}$ were 83 and 138 , respectively; thus, the quality factor of the LMW increases by $66 \%$ compared with that of the LCW. 


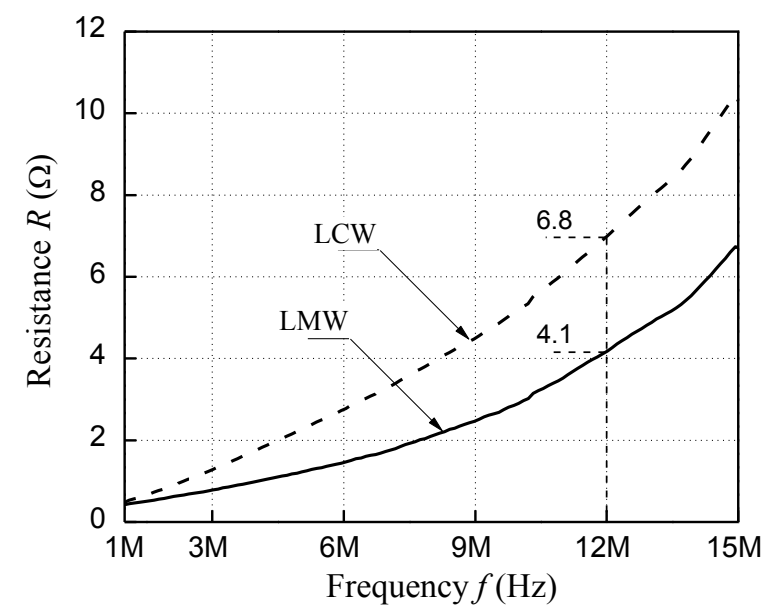

(a)

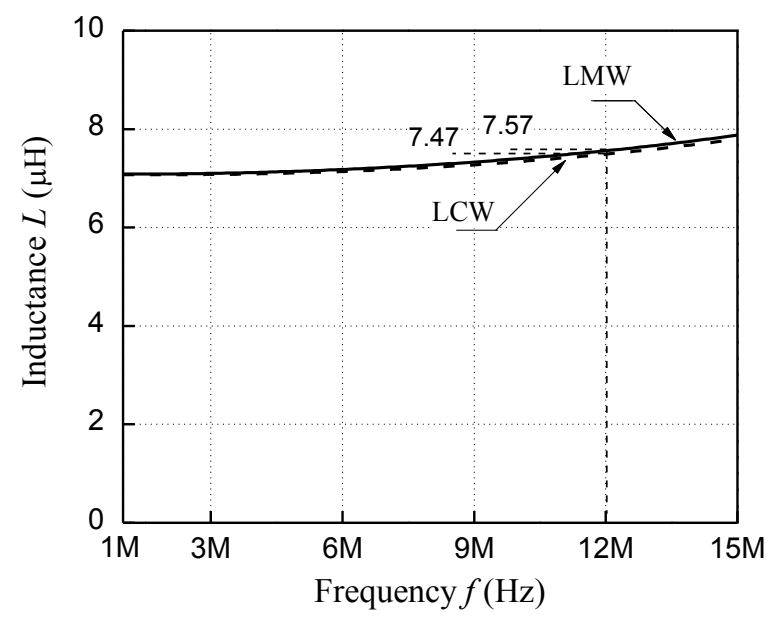

(b)

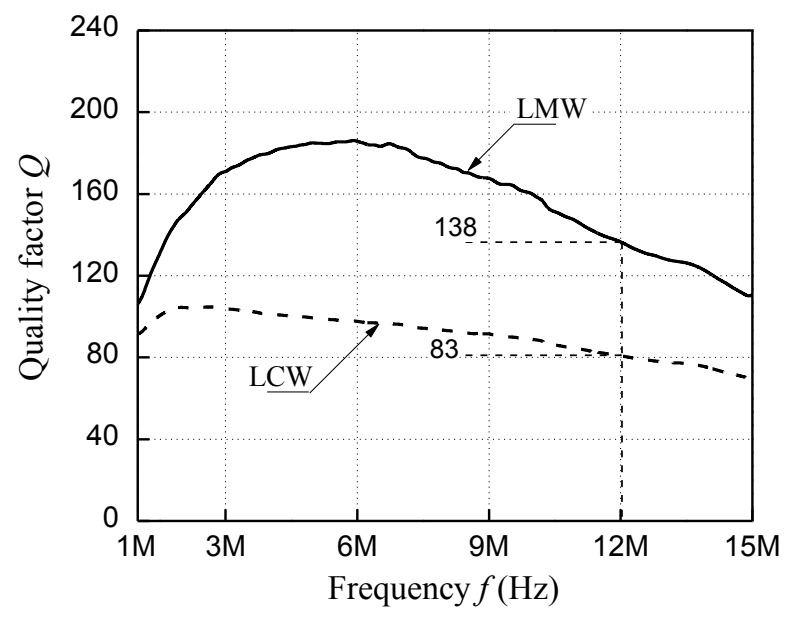

(c)

Fig. 5. Impedance characteristics of coils (measured with 4294A). (a) Resistance. (b) Inductance. (c) Quality factor.

Fig. 6 shows the coupling factor versus transmission distance characteristics. The coupling factors of the LCW and LMW coils at the transmission distance $l=18.5 \mathrm{~mm}$ were 0.093 and 0.091, respectively; thus, the coupling coefficient of the LMW

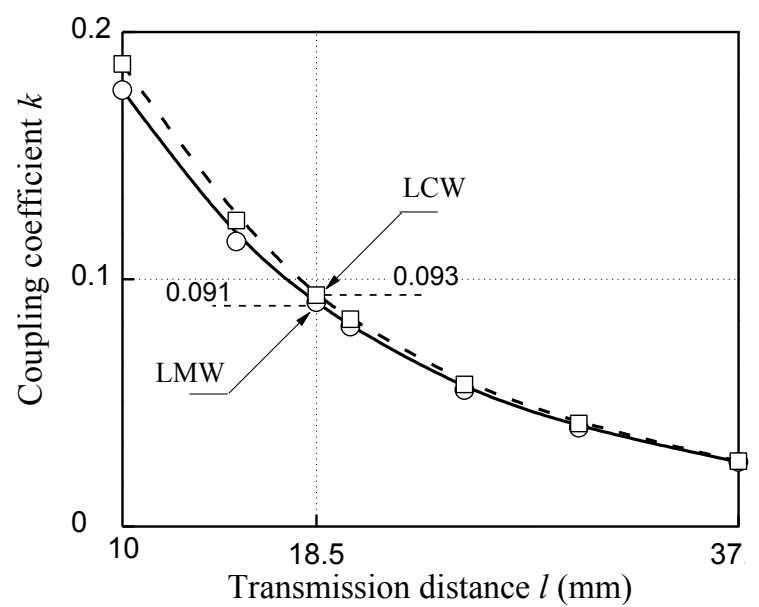

Fig. 6. Coupling coefficient characteristics (measured with 4294A).

decreases by $2.2 \%$ compared with that of the LCW. This is because the magnetic energy is stored in a magnetic thin film.

\section{TRANSMISSION EFFICIENCY CHARACTERISTICS}

\section{1) Efficiency characteristics}

Fig. 7 shows the transmission efficiency versus frequency characteristics of the LCW and LMW coils. The series resonance frequency of the LCW and LMW coils is approximately $13 \mathrm{MHz}$. The efficiency characteristics were measured with a network analyzer (Agilent, E5061B). The network analyzer is measured at $Z_{\mathrm{L}}=Z_{0}=50 \Omega$. The input power is $P_{\mathrm{i}}=0 \mathrm{dBm}$ in this measurement. Figs. 7 (a) and (b) show the transmission efficiency versus frequency characteristics of the coils at the transmission distances $l=10 \mathrm{~mm}$ and 18.5 $\mathrm{mm}$, respectively. In Fig. 7, $\eta_{11}$ is the reflection efficiency, and $\eta_{21}$ is the transmission efficiency. The maximum efficiencies $\eta_{21}$ of the LCW and LMW coils at the transmission distance $l=$ $10 \mathrm{~mm}$ were $69.8 \%$ and $77.7 \%$, respectively, indicating a $7.9 \%$ improvement in the efficiency of LMW coil. The efficiency $\eta_{21}$ also improves by $8.5 \%$ at the transmission distance $l=$ $18.5 \mathrm{~mm}$.

\section{2) Input impedance characteristics}

Fig. 8 shows the input impedance frequency characteristics of the coil connected to the capacitor $C_{\mathrm{r} 1}=C_{\mathrm{r} 2}=18 \mathrm{pF}$ in series. The receiving side is connected to the load impedance $Z_{\mathrm{L}}=50 \Omega$. Figs. 8 (a) and (b) show the input impedance versus frequency characteristics of the transmission distances $l$ $=10 \mathrm{~mm}$ and $18.5 \mathrm{~mm}$, respectively. The input impedance of the LCW and LMW coils at $l=10 \mathrm{~mm}, f=12 \mathrm{MHz}$ was both $Z_{\text {in }}=56 \Omega$ as shown in Fig. 8 (a). Therefore, the input impedance $Z_{\text {in }}$ is near the load impedance $Z_{\mathrm{L}}=50 \Omega$, and $\eta_{11}$ is $5 \%$ or less, and $\eta_{21}$ reaches the maximum value as shown in Fig. (7) (a). There is no reflection, and $\eta_{21}$ greatly depends on the ohmic loss of the coils. The improvement in the efficiency $\eta_{21}$ of the LMW coil originates from the increase in quality factor due to the reduction in $\mathrm{AC}$ resistance. It is similar to the above-mentioned for $l=18.5 \mathrm{~mm}$ as shown in Figs. 7 (b) and 8 (b). 


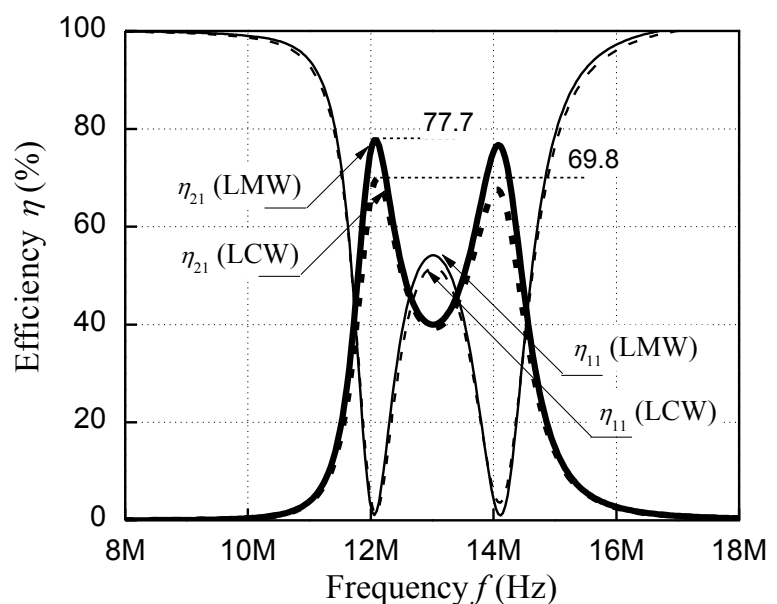

(a)

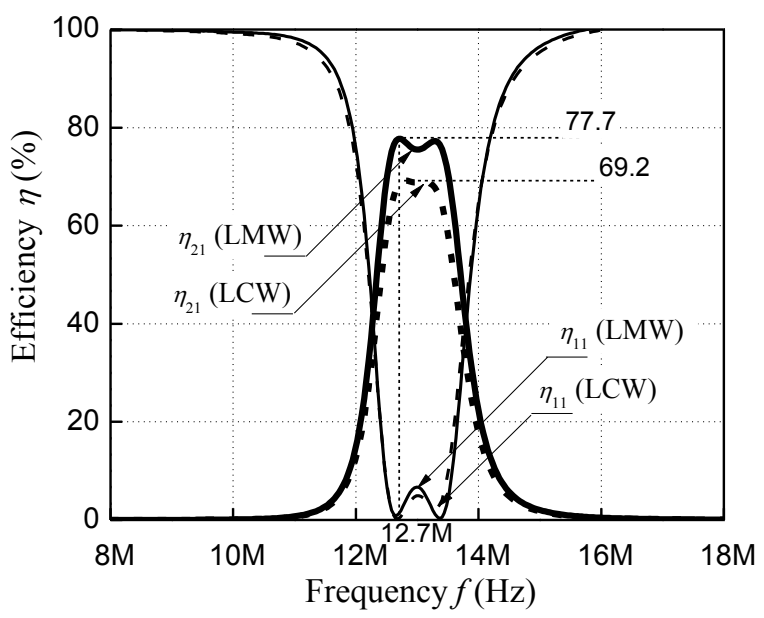

(b)

Fig. 7. Efficiency vs. frequency characteristics (measured with Agilent E5061B, $d_{\mathrm{i}}=37 \mathrm{~mm}, n=10, C_{\mathrm{r} 1}=C_{\mathrm{r} 2}=18 \mathrm{pF}$, $P_{\mathrm{i}}=0 \mathrm{dBm}, Z_{\mathrm{L}}=50 \Omega$ ). (a) $l=10 \mathrm{~mm}$. (b) $l=18.5 \mathrm{~mm}$.

\section{CONCLUSION}

\section{1) Impedance characteristics of coil}

The resistances of the LCW and LMW coils at the frequency of $12 \mathrm{MHz}$ were $6.8 \Omega$ and $4.1 \Omega$, respectively, thus, the resistance of the LMW decrease by $40 \%$ compared with that of the LCW. The quality factors of the LCW and LMW coils at the frequency of $12 \mathrm{MHz}$ were 83 and 138 , respectively; thus, the quality factor of the LMW increased by $66 \%$ compared with that of the LCW.

\section{2) Transmission efficiency characteristics}

The maximum efficiencies of the LCW and LMW coils at the transmission distance $l=10 \mathrm{~mm}$ were $69.8 \%$ and $77.7 \%$, respectively; thus, the efficiency of the LMW coil was improved by $7.9 \%$. The maximum efficiencies of the LCW and LMW coils at a transmission distance $l=18.5 \mathrm{~mm}$ were $69.2 \%$ and $77.7 \%$, respectively, the efficiency of the LMW coil improved by $8.5 \%$.

The above-mentioned characteristics originating from the $\mathrm{AC}$ resistance due to the proximity effect of the LMW decreased and the quality factor of the LMW improved.

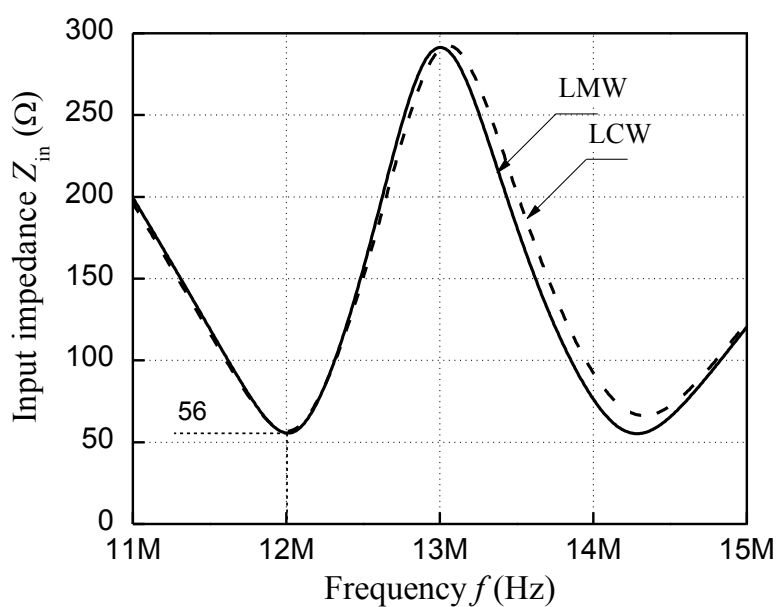

(a)

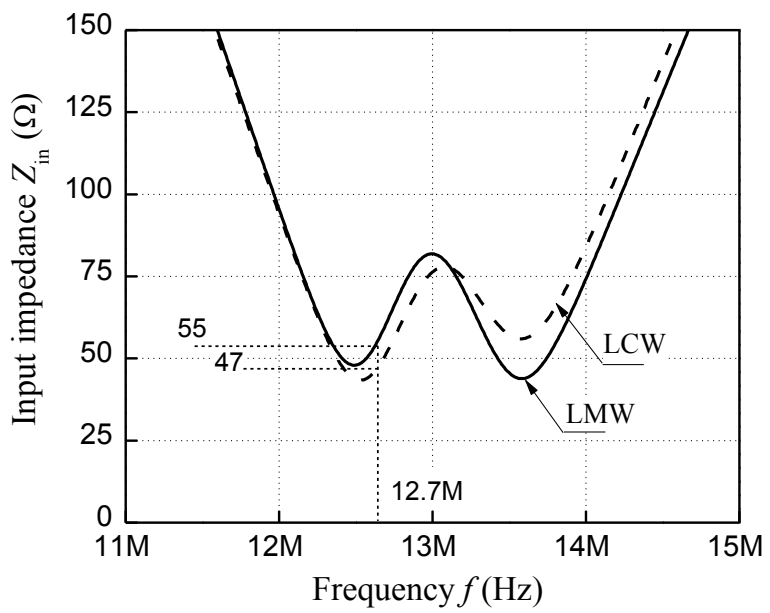

(b)

Fig. 8. Input impedance vs. frequency characteristics $\left(d_{\mathrm{i}}=37 \mathrm{~mm}, n=10\right.$, $C_{\mathrm{r} 1}=C_{\mathrm{r} 2}=18 \mathrm{pF}, Z_{\mathrm{L}}=50 \Omega$ ). (a) $l=10 \mathrm{~mm}$. (b) $l=18.5 \mathrm{~mm}$.

\section{REFERENCES}

[1] Y. Matsuda, H. Sakamoto, "Non-contact magnetic coupled power and data transferring system for an electric vehicle," J. Magn. Mater., Vol. 310, No. 2, Part 3, pp. 2853-2855, 2007.

[2] A. Karalis, et al., "Efficient wireless non-radiative mid-range energy transfer," Ann. Phys. Vol. 323, pp. 34-48, 2008.

[3] A. Jesus, A. Rafael, B. Jose M., B. Luis A., P. Diego, "Frequencydependent resistance in litz-wire planar windings for domestic induction heating appliances," IEEE Trans. Power Electron., Vol. 21, No. 4, pp. 856-866, 2006.

[4] T. Mizuno, S. Enoki, T. Hayashi, H. Shinagawa, "Extending the linearity range of eddy-current displacement sensor with magnetoplated wire," IEEE Trans. Magn., Vol. 43, No. 2, pp. 543-548, 2007.

[5] S. Yoshimura, S. Yoshihara, T. Shirakashi, E. Sato, K. Ishii, "Characteristics of high-Q coils composed of Fe-plated $\mathrm{Cu}$ wire," Magneto-Electronics International Symposium, pp. 485-487, 1994.

[6] T. Mizuno, S. Enoki, T. Asahina, T. Suzuki, M. Noda, H. Shinagawa, "Reduction of proximity effect in coil using magnetoplated wire," IEEE Trans. Magn., Vol. 43, No. 6, pp. 2654-2656, 2007.

[7] T. Imura, H. Okabe, T. Uchida, Y. Hori, "Study on open and short end Helical antennas with capacitor in series of wireless power transfer using magnetic resonant couplings" Annu Conf IEEE Ind Electron Soc., Vol. 35th Vol. 6 pp. 3654-3659, 2009. 